\title{
Shaken baby syndrome. Case report
}

\author{
Tatiana Iov ${ }^{2}$, Sofia David ${ }^{1}$, Simona Damian${ }^{1}$, A. Knieling ${ }^{1}$, \\ Mădălina Maria Diac ${ }^{1}$, D. Tabian², Diana Bulgaru-Iliescu ${ }^{1}$ \\ 1"Grigore T. Popa” University of Medicine and Pharmacy of Iași, Iasi Forensic Institute \\ ${ }^{2}$ Iasi Forensic Institute, ROMANIA
}

\begin{abstract}
Recognition of abuse and the treatment of child victims are recent concepts in the history of mankind. Increasing the awareness of the need to treat and prevent such abuse is a characteristic of modern society. The beaten child syndrome was described by Ambroise Tardieu in 1860, and Shaken Baby Syndrome (SBS) was clearly illustrated in medical literature a century later by Caffey in 1972. The definition of SBS is based on the association of major intracranial lesions with minimal external lesions and the diagnosis is still difficult to establish. The authors describe a reduced number of 7 cases of pediatric patients addressed for forensic expertise and where suspicion of SBS has arisen. The lesion mechanisms involved in the production of this syndrome are still controversial and are sources of frequent debates in legal medicine. These uncertainties can make legal punishment inoperable. The therapeutic management of these children in neurosurgery is not subject to international consensus, and discrepancies between different clinics impede a comparative cohort assessment. However, SBS is a major public health problem due to severe neurological injuries caused to child victims during brain development.
\end{abstract}

Key words: SBS, cerebral injuries

\section{Literature review}

The symptoms of the Shaken Baby Syndrome are difficult to identify, and the family usually avoid describing the cranial trauma suffered by the child. Nonspecific symptoms like vomiting, crying and irritability are often confused with gastric or intestinal disorders. Violent shaking is often repeated, but the one with the highest intensity is the one that causes the death. The macroscopic and microscopic examination of the brain, the examination of the eyes and bones can, in most cases, identify the Shaken Baby Syndrome.

The Shaken Baby Syndrome (SBS) is also called head trauma due to child abuse, shaking impact syndrome, or shaken child syndrome (8).

The syndrome of the shaken child was defined by Dr. Caffey, a radiologist, in 1972 (1) as a form of physical abuse to a 1-2 years old child. The most at risk are babies aged between 0 months and 2 years. The head of a baby is 
relatively heavy compared to his body, and the neck muscles are so weak that they cannot hold the baby's head very well (7). Shaking the baby can cause: blindness, brain damage, cerebral palsy, apoplexy attack, epilepsy, loss of hearing, learning difficulties, and later behavioral problems (9).

Does this syndrome occur only in children aged 1-2 years? No, in the United States, such cases have been reported in children up to 7 years old (2) and each year, about 1300 children are seriously injured or die from cranial trauma due shaking. (3)

Shaken child syndrome is a major cause of death, accounting for about $25-30 \%$ of all SBS children. Around $60-75 \%$ of the surviving children have mild or severe retardation, paralysis, including tetraplegia, cerebral paralysis, convulsive disorders and blindness.

The less serious cases of SBS are difficult to diagnose, especially in infants. A characteristic of the shaken child's syndrome is the absence of external traumatic signs, but the irritability, the slowed down rhythm of the growth, the slowing down of the deglutition reflex, lethargy, vomiting, depression, increase of head size, intracranial hypertension, changes in the respiratory system and dilated pupils are some of the signs. Other associated lesions are retinal bleeding, long bone fractures and subdual hematomas. $(4,5)$ Brain injuries of a traumatic nature, in children, without an external head injury, strongly advocate the shaking mechanism. $(2,6)$

In California, almost all SBS victims who survive are transferred to maternal care after diagnosis. In the United States, there is also a child-shatter prevention project that in the
West of New York has reduced the baby's syndrome rate by $55 \%$ and it is currently known as a national model. After the birth of the child, all parents were shown in the hospital a video recording about SBS prevention that includes simple information on how to act if the children cry and then they were asked to sign a commitment. This project has been replicated in several countries due to its resounding success. New York state's laws now require all hospitals to provide this program to all new parents. In recent years, some US states have passed a series of laws on the prevention of SBS (4).

\section{Etiology}

SBS is produced by vigorously shaking the child's body through his arms, legs, chest or shoulders. The child's head will thus perform repeated flexion-extension movements. It is a non-impact traumatic association, produced by an indirect whiplash mechanism, with repeated and accelerated decelerations, with snap back and forth head movements. The most dangerous are the rotational movements, which cause uneven and asymmetrical landslides of the cerebral hemispheres relative to each other in relation to the skull. However, there are authors who do not recognize the veracity of this mechanism and who consider that such injuries cannot be produced by simply shaking the baby's body, in the absence of an even minor impact between the cephalic extremity and a hard body/ surface. Obviously, there is also SBS with head impact, but in these cases, the lesions are somewhat specific to classical mechanical trauma mechanisms. Nevertheless, most of the literature in this 
domain recognizes and supports the existence of pure SBS without impact. The high sensitivity of children under 1 year old to such movements is due to several factors:

- the relatively large dimensions and mass of the cephalic extremity comparing to the rest of the body;

- the immaturity of the neck muscles, which cannot yet support the baby's head, allowing broad movements, which are further amplified by inertia, and without defense reflections to counteract the amplitude of the movements through muscle contractions;

- the space between the brain and the dura mater is large, allowing the brain to slip more freely in relation to the bone and meningeal structures;

- high water content of the child's brain compared to an adult's one;

- incomplete myelination of the brain nerve substance.

\section{Pathophysiology and morphopathology}

Because of shaking the baby's body, considering the morphological characteristics of the small child and the rigidity of intracranial fibrous structures (dura mater, falx cerebri, tentorium cerebelli), and the relatively fixed structures (cranial nerves, carotid blood vessels, or vertebrobasilar system) the brain will move inside the skull, with the result of characteristic lesions.

External findings are very poor, except when other forms of physical abuse are associated to SBS. However, it is possible to identify ecchymotic patches of the fingers of the aggressor on the victim's anterior and posterior chest, in the positions of hand holding. Under the conditions of a usual grip of the aggressor's hands on the child's chest, we may find symmetrical lesions, an oval ecchymosis on each of hemithorax region, anterior (by compression with the thumb) and multiple ecchymosis on the posterior thorax, paravertebral (compression with the other fingers). However, we should not forget that SBS can also be caused by shaking of the upper or lower limbs or shoulders, and can therefore find mild contusion (ecchymosis) at different levels. It is also possible to find external lesions of different age.

The internal exam detects some typical lesions, which grouped, define SBS:

- subdural hematoma is found in over $90 \%$ of cases. It occurs by rupture of the bridging veins as a result of the violent shaking, causing the head to snap back and forth on the neck. Bilateral and interhemispheric collections are frequently found. In many cases the volume of the hematoma is not very high, in contrast to the patient's severe clinical condition, which makes us to believe that in SBS the subdural hematoma would not be a cause of death. Although Caffey described chronically subdural hematoma as typical, modern literature reports the highest frequency for the occurrence of acute bleeding. Sometimes subdual hematomas of different age may coexist, indicating repeated trauma. Geddes mentions the appearance of the intradural hemorrhage as an effect of bridging vein rupture in SBS. In many cases, the subdural hematoma is accompanied by subarachnoid hemorrhages, with elective locations on the convexities of the cerebral hemispheres and their internal faces. 
- retinal hemorrhages are present in 85$100 \%$ of cases. Pre-retinal, intraretinally, and retroretinin hemorrhages, often confusing, are the most common; vitreous hemorrhages are rarely encountered (10\%). Retinal detachment can appear. In many cases (81-85\% of cases with retinal hemorrhages), the lesions are bilateral. It has not been established a relation between the location of retinal hemorrhages (right/left) and subdual hematomas. To explain these injuries, several theories have been issued:

- optical nerves shearing by the brain that slips inside the skull, for this mechanism advocating the relatively frequent findings of concentric retinal hemorrhages around the papilla of optic nerve;

- hypertension in the retinal venous system as a result of cerebral edema, but this is rather the pathophysiological explanation of Terson syndrome;

- vascular mechanism: Transient stop of blood flow due to compression of the vascular carotid-ophthalmic system during head shaking, or retinal vasospasm;

- Retinal hemorrhages as a result of inertial movements of the vitreous body during acceleration / deceleration cycles - Mechanism revealed in the small child due to its high vitreous consistency and strong adhesion to the vitreoretinal surface;

- vascular ruptures due to the slippage of the retinal layers and their disruption;

- acute thoracic compression with Valsalva effect and retinal hemorrhage due to increased intracerebral venous pressure.

In the ocular sphere, in the fatal cases, there are necroptic findings of bleeding around the optic nerves and axonal ruptures. Probably these lesions are common in SBS, being the cause of evolution with optic nerve atrophy in many cases where victims survive, the necroptic exam of a case revealed bilateral retro-ocular hemorrhages, but they may have been produced by local extension from the optic nerve sheaths presenting bleeding sleeves.

- parenchymal brain injury. Cerebral contusion appears quite rare due to the morphological immaturity of the brain structures, with the lack of myelinization. Contusive hemorrhagic lesions can occur in the corpus callosum due to its torsion during movements with the angular accelerator component, the basal faces of the frontal and temporal lobes, by contact with the rumpus surface of the skull base and the upper cerebellum pedunculum by striking the tentorium cerebelli. Meningo-cerebral lesions are usually accompanied by moderate brain edema. Diffuse axonal rupture is a severe and typical lesion for SBS, possibly involved more than the subdural hematoma in the infaust prognosis of the syndrome. These occur as a result of the rotating movements of the head, which create shear forces. Axonal ruptures are largely responsible for the clinical condition of the victim, with varying levels of mental status, and often even by the patient's death. As a technique for identifying these lesions in the histopathology laboratory, $68-\mathrm{kDa}$ neurofilament immunoprecipitation and beta amyloid precursor protein (beta-APP) have already been established, thus visualizing axonal amputation bulbs in the form of round or oval acidophilus masses. The technique can 
also be used to find axonal lesions in the optic nerves.

- Medullary lesions: subdural hematomas and high cervical medullary contusions are reported due to shaking of the victim's head. Axonal lesions were found. Periadventitial hemorrhage in the vertebral artery between $\mathrm{C} 1$ and C4 was reported by Gleckman in a SBS case. There are injuries that occurs through an indirect mechanism of gripping: rib fractures with typical paravertebral localization, scapular fractures, vertebral spine apophyses and long bone fractures in exceptional situations.

\section{Clinical diagnosis}

Upon reaching the hospital, patients with SBS typically have nonspecific symptoms, varying and sometimes progressive degrees of consciousness, and respiratory disturbances. The Glasgow Coma Scale (GCS) score is one of the most important indicators of the severity of the traumatic brain involvement in SBS. Data on the average Glasgow score in patients with SBS at the time of the arrival at the emergency unit varies depending on the author, in most cases ranging between 9-12 GCS. Thus, there is a correlation between the severity of the GCS and prognosis.

Gillilanda has made a definition of SBS based on a clinical diagnostic criterion: echimotic compression marks with fingers and/or ribs fractures, subdural and/or leptomeningeal hemorrhage and a history of vigorous shaking. According to this author, if at least two criteria are met, the diagnosis of SBS can be concluded. For ocular lesions, the diagnosis is primarily based on an exam of the bottom of the eye. It must, therefore, enter a mandatory protocol to recommend an ophthalmologic examination to a child under 1 year who presents a subdural hematoma, even in the absence of external signs of violence. A morphometric analysis of the retinal bleeding is also useful, as there has been observed a correlation between the retinal hemorrhage and the reality of SBS and visual prognosis.

\section{Paraclinical diagnosis}

The main investigations that contribute to the diagnosis of SBS are: Cranio-cerebral computer tomography (CT), nuclear magnetic resonance imaging (MRI), high-resolution cranial ultrasound, thoracic radiography (for the diagnosis of possible costal fractures), laboratory blood tests (for the differential diagnosis of hemorrhagic lesions) and lumbar puncture.

\section{Forensic aspects}

In case of the survival of the victim, the forensic physician will have to interpret the medical documentation prepared by other specialists (pediatricians, neurosurgeons, ophthalmologists) and in collaboration with them, to make a synthesis and establish the diagnosis of SBS. It should be kept in mind:

- The presence of lesions considered as specific to the syndrome (subdural hematoma, retinal hemorrhage);

- The exclusion of other violent or nonviolent causes responsible for causing such injuries; 
- The exclusion of traumatic head injury, following a very careful external examination;

- A history of violent shaking of the child's body.

- The time of medical care for healing of SBS is around 45-50 days but, in some cases, it may exceed 60 days, endangering the life of the victim through the existence of the subdural hematoma.

- The legal framing of the deed is also given by the intentional element, as, in most of cases the perpetrator did not wish to produce these consequences. Sometimes he/she did not even know that shaking the child with the intention to make him stop crying can cause such serious injuries.

- In the case of healing with sequelae, the forensic physician will appreciate the existence of a physical or mental disability, the loss of a function (visual, auditory) and the level of functional deficit created because of the trauma.

In cases of death of the victim, the forensic physician will not see, during an external examination, any signs or lesion due to violence, but he will discover serious internal lesions. As a rule, in all cases where a subdural hematoma is identified in a baby child, it is mandatory to examine and collect the eyeballs (through the endocranial orbital approach) for microscopic examination. It is recommended to examine the brain after putting it in $10 \%$ formalin for 3 weeks, to better identify the traumatic brain injuries. The examination of the fresh sample and its sectioning are difficult and predisposes to artifacts due to the low consistency of the brain in children of this age.
- The microscopic examination is mandatory to confirm the macroscopicallyobserved meninges, cerebral and ocular lesions, to diagnose the microscopic traumatic lesions, to attest their vital lesion character and to assess its age.

- fragments of cerebral substance from all areas with macroscopic lesion will be drawn. For the immunohistochemistry, samples from the white substance of both cerebral hemispheres, cerebellum, cerebral trunk, marrow, optic nerves, will be collected. Usually, staining with hematoxylin-eosin is used, but Perls staining is also useful in assessing the age of a hemorrhagic lesion because it highlights hemosiderin.

- The microscopic examination of the cerebral and medullary substance, and of the cranial nerves after immune marker for the beta-amyloid precursor protein (beta-APP) or for neurofilament (68-kDa neurofilament), positive techniques for axonal ruptures, but still hardly accessible in the forensic services in our country.

- The eyeballs will be examined after fixation in formalin and sagittal sections will be practiced, one of them continuing with longitudinal sectioning of the optic nerve.

\section{Case report}

The emergency sheet revealed that the child was hospitalized for 35 minutes. At the hospital admission the child was having first degree coma, left eye = right eye, fixed mydriasis, corneal and deglutition reflexes were present, free airways, spontaneous breathing, physiological vesicular murmur, absent rales, cardiac sinusal rhythm, 80 beats 
per minute heart rate. The craniocerebral CT scan revealed a parenchymatous hypodensity extended on the entire left hemisphere and also on the right frontal lobe paramedian on the right anterior carotid artery's territory, significant collapse of the left lateral ventricle, subfalcine engagement of the median line structures, bilateral transtentorial engagement.

A copy of the consultation sheet showed that the child was hospitalized for 17 hours and 44 minutes with the diagnosis of" Massive cerebral infarct in the territory of the medium carotid artery, posterior carotid artery, left anterior carotid artery and right anterior carotid artery". The patient's reason for presentation: third degree coma, bilateral fixed mydriasis. The medical history showed that the child developed consciousness disorders apparently after a trauma through aggression. Neurological examination: without active movements, areflexia (hyporeflexia), reactive exclusively to pain. Local examination: right frontal localized excoriations at the internal and external angle of the left eye and at the left auricular concha; right laterocervical localized ecchymosis at thighs and buttocks bilateral. From the epicrisis of the observation sheet it turned out that the evolution was unfavorable with the installation of a non-reversible cardiorespiratory arrest at the resuscitation maneuvers and exitus.

\section{Necroptic exam revealed:}

There was no investigation data at the time of the necropsy. After a through investigation of social data it appears that the minor was in the care of his grandmother with his other brothers. It also appears that the grandmother repeatedly applied "physical corrections" to him, striking him with the hands or hitting him with a strap. The last correction was applied by taking him to his shoulders and shaking him, then applying several strokes with her hand over baby's head and body, then hit him with a rubber hose (for household use). Immediately after this correction, the minor felt dizziness, lethargic, unable to keep his balance, reason why her grandmother sit him in the bed. Six hours after the "correction", the child, who was sitting on the bed, fell aside, fed an insufficiently, drank water, and then fell asleep. Two days after the "correction", the condition of the child got worse and the ambulance was announced.

The body belongs to a male child, $89 \mathrm{~cm}$ in height, age-appropriate constitution, 2 years and 10 months old, with a known identity.

Macroscopically examination revealed multiple scratches: on the right frontal region, left eye internal and external angle, mandibular- left side, left laterocervical region; multiple ecchymosis with localization on the forearm and left hand, at the buttocks, thighs and calves. Internal examination findings were: left hemisphere subdural hematoma 0.4 $\mathrm{cm}$ thick; cerebral edema; contusion and cerebral dilaceration at the base of left cerebral hemisphere.

The anatomo-pathological microscopic examination establishes the following: focal inflammatory infiltration in leptomeninges, diffuse cerebral contusion, important edema in perivascular and pericelular spaces.

Due to the absence of high-intensity external cranial marks and the presence of traumatic brain injuries, in the provisional 
conclusions, the mechanism of production was established as a mechanism for shattering.

Later on, the mechanism for brain lesions (the subdural hematoma, the contusion and the cerebral dilaceration) was issued: it was possible to produce it most probably by vibrating cerebral substance in the sudden mobilization of the child's body and head by another person, but it cannot be excluded repeatedly active blows to the head with or by blunt objects, if the investigation demonstrates this mechanism.

The grandmother was assessed by forensic experts and psychiatrists. At the examination of the abuser it was concluded that the grandmother suffers from major depressive disorder with anxious phobic elements and preinvolutive background with reactive onset. The grandmother's behavior creates the constitutive elements of the first degree murder and has been committed with discernment.

\section{Conclusions}

The mechanism of producing craniocerebral lesions was most likely a hyperflexion followed by head hyperextension due to trauma to the neck that interested the left carotid artery; clarification of the mechanism was done with neurosurgeon specialists.

Neurosurgeon specialists have hypothesized about the WHIPLASH INJURY cranio-cerebral lesion mechanism, which can explain brain contusion lesions associated with cerebral infarction lesions as a result of repeated traumatic lesions of the left carotid artery at the cervical level.
Because this type of trauma is produced as a result of lack of education in this respect, we appreciate that prevention measures are of paramount importance, with physicians taking the lead in these actions. Consequently, even in maternity, doctors and nurses need to inform the parents of the little boy barely come into the world about the adverse consequences of shaking the child. Massive campaigns over time in civilized countries, supported by the media, had the motto: "never shake the child!". Guidelines for parents are simple messages that aim to summarize the attitude to adopt in difficult times.

Because this type of trauma is produced as a result of lack of education in this respect, we appreciate that prevention measures have importance, doctors has the main role in these actions. Therefore, even in maternity, doctors and nurses have to inform the parents of the little boy barely come into the world about the adverse consequences of shaking baby.

\section{Correspondence}

Anton Knieling

tony_knieling@yahoo.com

\section{References}

1. Caffey J. On the theory and practice of shaking infants. Its potential resitual effects of permanent brain damage and mental retardation. Am J Forensic Med Pathology. 1972;22:112-122.

2. Salehi-Had H, Brandt JD, Rosas AJ, Rogers KK. Findings in older children with abusive head injury: does shaken-child syndrome exist. Pedriatics Journal. 2006;117:1039-1044.

3. Keean $\mathrm{H}$ et al. A Population-Based Study of Inflicted Traumatic Brain Injury Children. JAMA, The Journal of the American Medical Association. Vol. 290. 2006;290(5):621-626. 
4. Dias M et al. Preventing Abusive Head Trauma Among Infants and Young Children: A Hospital - Based, Parent Education Program. Pediatrics: Official Journal of the American Academy of Pediatrics. 2005;115(4):470-477/

5. Ioan BG. Tanatologie medico-legală - note de curs. Editura Junimea. Iaşi. 2007, p 55-56.

6. Grigoriu C. Repere în patologia medico-legală. Editura Junimea. Iaşi. 2007, p.135.
7. Plăhteanu M, Baciu Gh, Pădure A. Aspecte medicolegale lezionale în traumatologia mecanică. Editura Performantica. Iaşi. 2004, p.164.

8. Ciurea AV, Davidescu HB: Traumatologie craniocerebrală. Editura Universitară „Carol Davila”. Bucureşti. 2007, p. 287.

9. Bulgaru-Iliescu D. Anomia microsocială - forme şi consecinţe. Editura Timpul. 2002, p 117-118. 